\title{
Tratamento cirúrgico da endocardite em prótese valvular cardíaca
}

Pablo M. A. POMERANTZEFF*, Paulo M. PÊGO-FERNANDES*, Yukio KIOKA*, Rita H. A. CARDOSO*, Silvana D. D. GALUCCI*, Alfredo MANSUR*, Altamiro Ribeiro DIAS*, Max GRINBERG*, Delmont BITTENCOURT*, Noedir A. G. STOLF*, Geraldo VERGINELLI*, Adib D. JATENE*

RBCCV 44205-76

POMERANTZEFF, P. M. A.; PÊGO-FERnANDES, P. M.; KIOKA, Y.; CARDOSO, R. H. A.; GALUCCI,

S. D. D.; MANSUR, A.; DIAS, A. R.; GRINBERG, M.; BITTENCOURT, D.; STOLF, N. A. G.; VERGINELLI,

G.; JATENE, A. D. - Tratamento cirúrgico da endocardite em prótese valvular cardiaca. Rev. Bras.

Cir. Cardiovasc., 4(1): 43-50, 1989.

RESUMO: No período de janeiro de 1983 a março de $1988,1.512$ pacientes foram submetidos a substituição valvar, no Instituto do Coração, sendo 28 (1,85\%) deles por endocardite em prótese valvular. Dezessete doentes eram do sexo masculino e a idade variou de 18 a 67 anbs, com média e desvio padrāo de $36,7 \pm 12,9$. A avaliação da classe funcional (C.F.) revelou 11 pacientes em C.F. IV, 12 em C. F. III e 5 em C.F. II. Oito $(28,5 \%)$ pacientes foram operados em condiçōes de emergência. Um paciente era portador e válvula mecânica e 27 , de válvula biológica. As hemoculturas foram positivas em $14(50 \%)$ pacientes; o agente mais encontrado foi o Streptococcus viridans em 5 casos. 0 ecocardiograma realizado no pré-operatório em 27 pacientes mostrou correlação com os achados cirúrgicos em $26(96,2 \%)$. Na cirurgia, 17 doentes apresentavam vegetação na prótese e 11, abscesso no anel. Na retroca, foram utilizadas biopróteses em 27 (96,4\%) pacientes. A mortalidade imediata foi de 28,5\% ( 8 doentes), ocorrendo 1 óbito tardio. A análise da associaçăo óbito e tempo de aparecimento da endocardite, condição cirúrgica e resultado da cultura foi feita pelo teste de Qui-Quadrado $\left(\chi^{2}\right)$. Podemos concluir que as condiçōes clínicas pré-operatórias interferem, decisivamente, no resultado cirúrgico; a cirurgia de emergência tem resultados piores, devido às condiçōes mais críticas dos doentes; as endocardites mais precoces são mais graves; a manipulação de focos infecciosos em pacientes com prótese valvular deve ser cuidadosa e precedida de antibioticoterapia e, nos doentes sobreviventes à operação, a evolução a longo prazo apresenta melhora significativa da classe funcional.

DESCRITORES: endocardite, cirurgia; próteses valvulares cardiacas, cirurgia.

\section{INTRODUÇĀO}

A endocardite em próteses valvulares (EPV) é complicação pouco freqüente, porém muito grave. A intervenção cirúrgica é, freqüentemente, necessária, porém há controvérsia em relação ao melhor momento para a indicação. Diversos autores $1,2,4,7,16$ demonstram melhores resultados quando o paciente está controlado clinica- mente, porém, na maioria dos pacientes, a cirurgia é realizada em condições gerais muito graves, por impossibilidade absoluta de controle adequado.

O objetivo do presente trabalho é a análise de 28 pacientes operados, consecutivamente, no Instituto do Coração, no período de janeiro de 1983 a março de 1989, por endocardite em prótese valvular.

Trabalho realizado no Instituto do Coração do Hospital das Clínicas da Faculdade de Medicina da Universidade de São Paulo. Săo Paulo, SP. Brasil.

* Do Instituto do Coração do Hospital das Clínicas da Faculdade de Medicina da Universidade de São Paulo.

Apresentado ao 16: Congresso Nacional de Cirurgia Cardíaca. São Paulo, SP, 7 e 8 de abril, 1989.

Endereço para separatas: Pablo M. A. Pomerantzeff. Av. Dr. Enéas Carvalho de Aguiar, 44 -Divisăo Cirúrgica. 05403 Săo Paulo, SP, Brasil. 
POMERANTZEFF, P. M. A.; PÊGO-FERNANDES, P. M.; KIOKA, Y.; CARDOSO, R. H. A.; GALUCCI, S. D. D.; MANSUR, A.; DIAS A. R.; GRINBERG, M.; BITTENCOURT, D.; STOLF, N. A. G.; VERGINELLI, G.; JATENE, A. D. - Tratamento cirúrgico da endocardite em prótese valvular cardiaca. Rev. Bras. Cir. Cardiovasc., 4(1): 43-50, 1989.

\section{CASUISTICA E MÉTODOS}

No período de janeiro de 1983 a março de 1988 , 1512 pacientes foram submetidos a substituiçāo valvar, no Instituto do Coração, sendo 714 trocas de valva mitral, 631 de valva aórtica, 130 duplas trocas mitro-aórticas e 37 substituições em posições diversas. Vinte e oito $(1,58 \%)$ doentes tiveram como indicação a endocardite em prótese valvular, sendo 12 em posição mitral, 11 aórtica e 5 mitral e aórtica.

As idades dos 28 pacientes variaram de 18 a 67 anos, com média e desvio padrão de $36,7 \pm 12,9$. Dezessete $(60,7 \%)$ doentes eram do sexo masculino e a cor branca ocorreu em $26(92,8 \%)$.

A febre foi o sintoma mais freqüente, ocorrendo em $27(96,4 \%)$ pacientes. No Gráfico 1, detalhamos a ocorrência de cada sintoma por grupo acometido. A avaliação da classe funcional (C. F.) (NHYA) revelou 11 pacientes em C. F. IV, 12 em C. F. III e 5 em C. F. II.

\section{GRÁFICO 1}

ENDOCARDITE EM PRÓTESE VALVULAR QUADRO CLÍNICO

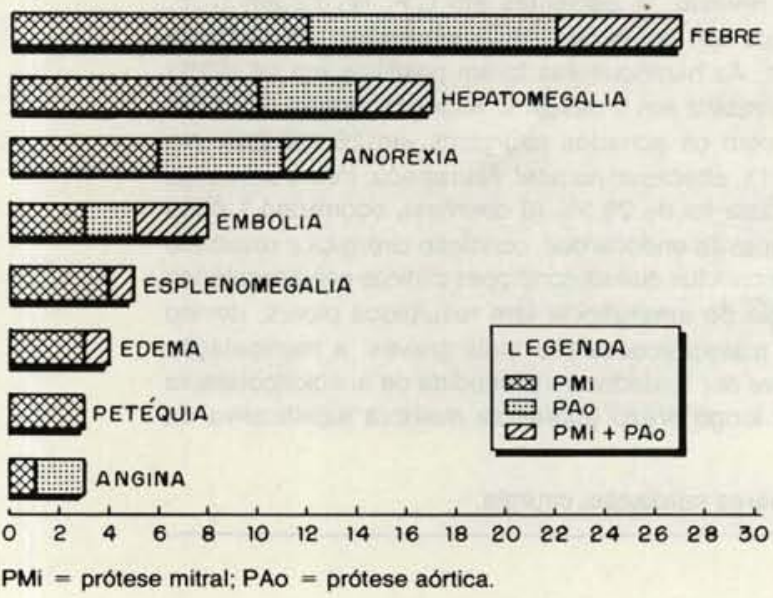

O hemograma revelou leucocitose em 17 pacientes e anemia em 9. Insuficiência renal foi encontrada em 10 doentes, hematúria em 8 , alteraçōes importantes do coagulograma em 4 e insuficiência hepática em 3 (Gráfico 2).

Em todos os pacientes foram colhidas amostras de sangue para hemocultura; em 27 doentes foi realizado ecocardiograma pré-operatório e, em 6 , estudo angiográfico.

Foi realizado o teste Qui-Quadrado $\left(\boldsymbol{x}^{2}\right)$ de Pearson (FLEISS ${ }^{14}$ ) para avaliar a associação entre o óbito e os fatores: tempo decorrido entre a troca valvar e o aparecimento da endocardite, resultado da hemocultura e condição cirúrgica (eletiva ou emergência).

\section{GRÁFICO 2}

ENDOCARDITE EM PRÓTESE VALVULAR

Testes Laboratoriais

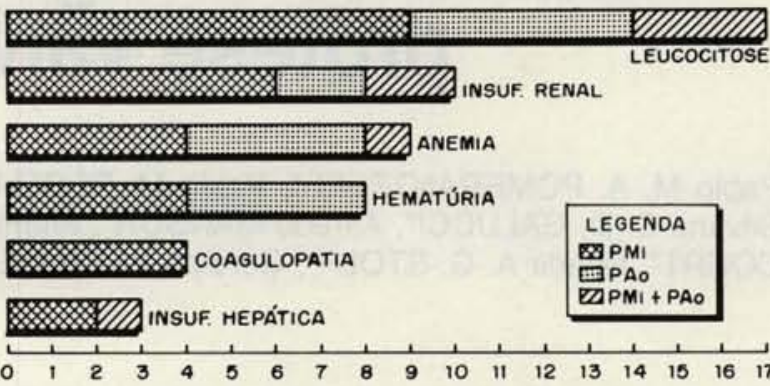

$\mathrm{PMi}=$ prótese mitral; $\mathrm{PAO}=$ prótese aórtica. Os diagnósticos de insuficiência renal $e$ insuficiência hepática foram obtidos através de provas laboratoriais, sendo, por esse motivo, incluidos nesta tabela.

Oito pacientes foram operados em condição de emergência, sendo 4 com próteses mitrais, 2 com aórticas e 2 com mitro-aórticas. Dos 4 mitrais, 2 foram operados em edema agudo dos pulmōes, 1 em choque séptico, e outro por embolia. Dos 2 doentes com prótese aórtica, 1 por hemólise e outro por choque séptico. Os 2 pacientes com EPV operados de emergência encontravam-se em choque cardiogênico.

Dados epidemiológicos do desenvolvimento da EPV foram: 3 pacientes tiveram complicaçāo de focos infecciosos dentários sem antibioticoterapia profilática, tendo o primeiro hemocultura positiva para St. epidermidis e os outros 2, negativa. Três doentes apresentaram infecções de vias biliares: 1 pneumonia; 1 infecção puerperal pós-aborto séptico com realização de histerectomia; 1 diverticulose de cólon com hemocultura para $S t$. bovis.

Com relação às próteses mitrais infectadas, 5 eram de dura-máter com aparecimento tardio da endocardite (acima de 4 anos após a operação) e 7 eram de pericárdio bovino. Em relaçăo às próteses aórticas, 3 eram heterólogas de porco, 1 de pericárdio bovino e 1 de Starr-Edwards (Tabela 1).

\section{RESULTADOS}

As hemoculturas foram positivas em $14(50 \%)$ pacientes (Tabela 2). Os agentes mais encontrados foram o Streptococcus viridans em 5 casos, Staphylococcus epidermidis em 2 e gram-negativos em 2; todos esses apresentaram EPV tardiamente (acima de 1 ano após a operação). Na EPV precoce (abaixo de 1 ano), apenas 2 pacientes apresentaram hemoculturas positivas, sendo um Corynebacterium JKe outro Staphylococcus aureus. 
POMERANTZEFF, P. M. A.; PÊgo-Fernandes, P. M.; KIOKA, Y.; CARDOSO, R. H. A.; GaluCCI, S. D. D.; MANSUR, A.; DIAS A. R.; GRINBERG, M.; BITTENCOURT, D.; STOLF, N. A. G.; VERGINELLI, G.; JATENE, A. D. - Tratamento cirúrgico da endocardite em prótese valvular cardiaca. Rev. Bras. Cir. Cardiovasc., 4(1): 43-50, 1989.

TABELA 1

TEMPO DECORRIDO ENTRE A TROCA VALVAR EA ENDOCARDITE

\begin{tabular}{crccc}
\hline & & $0-3 m$ & $3-12 m$ & $12 m$ \\
\hline MITRAL & DM & $3(2)$ & $5(1)$ \\
$n=12$ & PB & & 4 \\
& DM & & 3 \\
AÓRTICO & PB & $1(1)$ & & 3 \\
$n=11$ & PORCINA & $1(1)$ & $2(1)$ & \\
STARR & & 1 & \\
Mi + & DT & & $1(1)$ \\
AÓRTICO & PB & $2(2)$ & 1 & 1 \\
$n=5$ & PORCINA & & 1 \\
& TOTAL & $4(4)$ & $7(3)$ & $17(2)$ \\
\hline
\end{tabular}

$\mathrm{DM}=$ bioprótese de dura-máter; $\mathrm{PB}$ = bioprótese de pericárdio bovino; ( ) óbito; $m=$ meses.

TABELA 2

ENDOCARDITE E PRÓTESE VALVULAR CULTURAS

PMI PAO PMI +PAO TOTAL

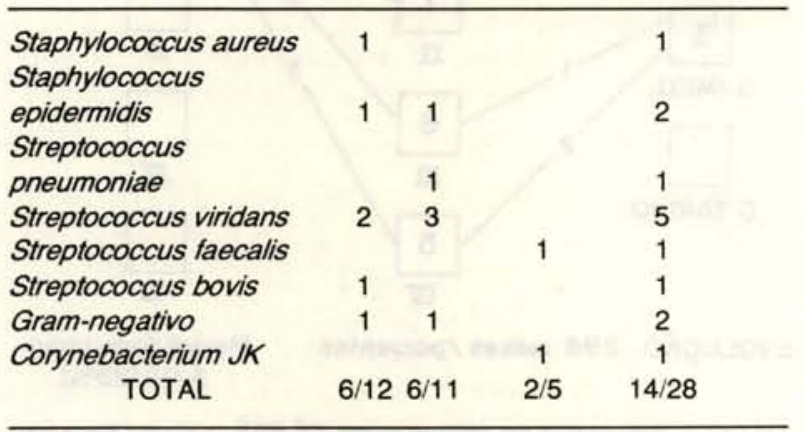

$\mathrm{PMi}=$ prótese mitral; $\mathrm{PAo}=$ prótese aórtica .

O ecocardiograma pré-operatório realizado em 27 pacientes mostrou, em 16, vegetaçōes na prótese, que foram confirmadas na cirurgia. Em 10 doentes nos quais o ecocardiograma não demonstrou vegetaçōes também não foram encontradas, na operação. No paciente em que o ecocardiograma não visibilizou vegetaçōes, mostrou a prótese espessada e, na cirurgia, encontraram-se vegetaçōes apenas discretas.

Quanto aos achados cirúrgicos, em 17 pacientes foram encontradas vegetações na prótese; em 11, abscesso no anel; em 4, calcificação da prótese; em 4, escape perivalvular e, em 2, fístulas (Gráfico 3).

$\mathrm{Na}$ retroca valvular, foram utilizadas biopróteses de pericárdio bovino em 7 pacientes aórticos, 9 mitrais e 3 mitro-aórticos. Biopróteses heterológas de porco foram usadas em 3 pacientes aórticos, 3 mitrais e 2 mitro-aór-

\section{GRÁFICO 3}

ENDOCARDITE EM PRÓTESE VALVULAR Aspecto cirúrgico

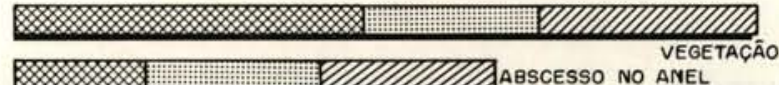

\begin{tabular}{|c|c|}
\hline UA CALCIFICAÇĀO & LEGENDA \\
\hline ESCAPE PARAVALVAR & 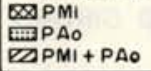 \\
\hline
\end{tabular}

\#\#ASTULA

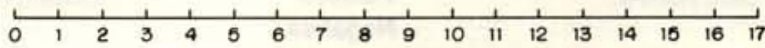

$\mathrm{PMi}=$ prótese mitral; $\mathrm{PAO}=$ prótese aórtica.

ticos. Uma prótese modelo Starr-Edwards foi colocada em 1 paciente aórtico.

Com relação aos procedimentos cirúrgicos associados, em 5 pacientes foi realizado tratamento cirúrgico de abscesso do anel aórtico, sendo que, em 1, foi utilizada placa de pericárdio bovino para a correção, em 1 paciente foi feita ampliação do anel aórtico; em 1, retirada do trombo de átrio esquerdo; em 1, plástica de valva mitral e, em 1, revascularizaçāo do miocárdio.

As principais complicaçōes pós-operatórias foram: baixo débito, septicemia e coagulopatia (Tabela 3 ).

A mortalidade imediata foi de $8(28,5 \%)$ pacientes, sendo que a mortalidade imediata, para os pacientes mitrais, foi de $3(25 \%)$ pacientes, para os aórticos, 2 $(27,2 \%)$ pacientes e, para os pacientes mitro-aórticos, em número de $2(40 \%)$.

Ocorreu 1 óbito tardio, correspondente a $5,4 \%$ dos sobreviventes.

A análise dos nossos resultados (Tabela 4) também demonstrou: 1) associaçāo entre tempo decorrido da

TABELA 3

COMPLICAÇOEES POSS-OPERATORIAS

\begin{tabular}{llllr}
\hline & $\begin{array}{c}\text { PMi } \\
n: 12\end{array}$ & $\begin{array}{c}P A O \\
n: 11\end{array}$ & $\begin{array}{c}P M i+P A O \\
n: 5\end{array}$ & TOTAL \\
\hline Reop. por sangramento & 1 & & & 1 \\
Coagulopatia & 5 & $3(1)$ & 4 & $12(1)$ \\
Baixo débito & 9 & $6(1)$ & $5(2)$ & $20(3)$ \\
Alteraçōes neurológicas & 5 & 2 & $1(1)$ & $8(1)$ \\
Pneumonia & 2 & 4 & & 6 \\
Insuf. renal & 3 & 3 & 2 & 8 \\
Insuf. hepática & 2 & & & 2 \\
Septicemia & $6(3)$ & $3(1)$ & & $9(4)$ \\
Embolia & 1 & & & 1 \\
BAVT & 2 & 2 & & 4 \\
\hline
\end{tabular}

$\mathrm{PMi}=$ prótese mitral; PAo $=$ prótese aórtica; $($ ) óbito. 
POMERANTZEFF, P. M. A.; PÊGo-FeRnANDES, P. M.; KIOKA, Y.; CARDOSO, R. H. A.; GALUCCI, S. D. D.; MANSUR, A.; DIAS A. R.; GRINBERG, M.; BITTENCOURT, D.; STOLF, N. A. G.; VERGINELLI, G.; JATENE, A. D. - Tratamento cirúrgico da endocardite em prótese valvular cardiaca. Rev. Bras. Cir. Cardiovasc., 4(1): 43-50, 1989.

TABELA 4

ÓBITO

\begin{tabular}{|c|c|c|c|c|c|}
\hline & & $N \overline{A O}$ & $S I M$ & & \\
\hline TEMPO & $\begin{array}{l}>1 \text { ano } \\
<1 \text { ano }\end{array}$ & $\begin{aligned} 4 & (14,3 \%) \\
15 & (53,6 \%)\end{aligned}$ & 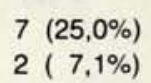 & 8,24 & 0,004 \\
\hline b) COND. CIRURG. & $\begin{array}{l}\text { Emergência } \\
\text { Eletiva }\end{array}$ & $\begin{aligned} 3 & (10,7 \%) \\
16 & (57,1 \%)\end{aligned}$ & $\begin{array}{l}5(17,9 \%) \\
4 \quad(14,3 \%)\end{array}$ & 4,73 & 0,030 \\
\hline c) CULTURAS & $\begin{array}{l}\text { Positiva } \\
\text { Negativa }\end{array}$ & $\begin{array}{r}12(42,9 \%) \\
7(25,0 \%)\end{array}$ & $\begin{array}{l}2(7,1 \%) \\
7(25,0 \%)\end{array}$ & 4,09 & 0,043 \\
\hline
\end{tabular}

Análise da associação entre o óbito e os fatores; a) tempo decorrido entre a troca valvar e o aparecimento da endocardite; b) condição cirúrgica; $c$ ) resultado da cultura. Utilizamos o teste Qui-Quadrado $\left(\chi^{2}\right)$ de Pearson para análise estatística.

troca valvar, aparecimento da endocardite e o óbito ( $p$ $=0,004)$ no sentido de se ter uma maior proporção de óbitos em pacientes cujo aparecimento da endocardite foi no 1: ano após a troca valvar; 2) associação entre condiçāo cirúrgica (emergência ou não) e óbito ( $p=0,030$ ), uma vez que se obteve maior proporção de óbitos em pacientes operados em condição de emergência. Além disto, 3 ) os resultados sugeriram associaçōes entre mortalidade e cultura negativa ( $p=0,043$ ).

No seguimento dos pacientes, 2 apresentaram novo episódio de endocardite na prótese, sendo 1 aórtico, que faleceu no $32^{\circ}$. dia de pós-operatório, e outro mitro-aórtico, com infecção das 2 próteses, aos 3 meses de evolução, após reintervençāo cirúrgica.

No seguimento ambulatorial, com tempo de acompanhamento de 708 meses/pacientes, os 18 sobreviventes encontram-se em classe funcional I (NHYA). Os 9 pacientes mitrais sobreviventes tiveram tempo de acompanhamento de 296 meses/pacientes, os 8 aórticos 300 meses/pacientes e os 2 mitro-aórticos, 112 meses/ pacientes (Gráficos 4, 5 e 6).

\section{DISCUSSÃO}

A endocardite em prótese valvular é complicação pouco freqüente, variando de 2,4 a $4,4 \%$ das trocas valvares $^{2}, 3,4,9,11$. A sua gravidade, porém, faz com que os vários autores revejam periodicamente a sua experiência.

A suspeita diagnóstica da endocardite em prótese é feita através do quadro clínico. A febre tem sido $\odot$ sintoma mais freqüente, tanto na nossa experiência $(96,4 \%)$, como na de outos autores ${ }^{5}$. Outros sinais comuns são presença de sopros, insuficiência cardíaca congestiva, embolias para múltiplos órgãos e esplenomegalia $^{1-16}$. A hemocultura é exame de grande valia na confirmação diagnóstica, sendo positiva em até $99 \%$ dos casos $^{5}$. A maioria dos autores $1,4,7,11,12,15$ cita como agente mais freqüente o estafilococo ${ }^{3,16}$, especialmente

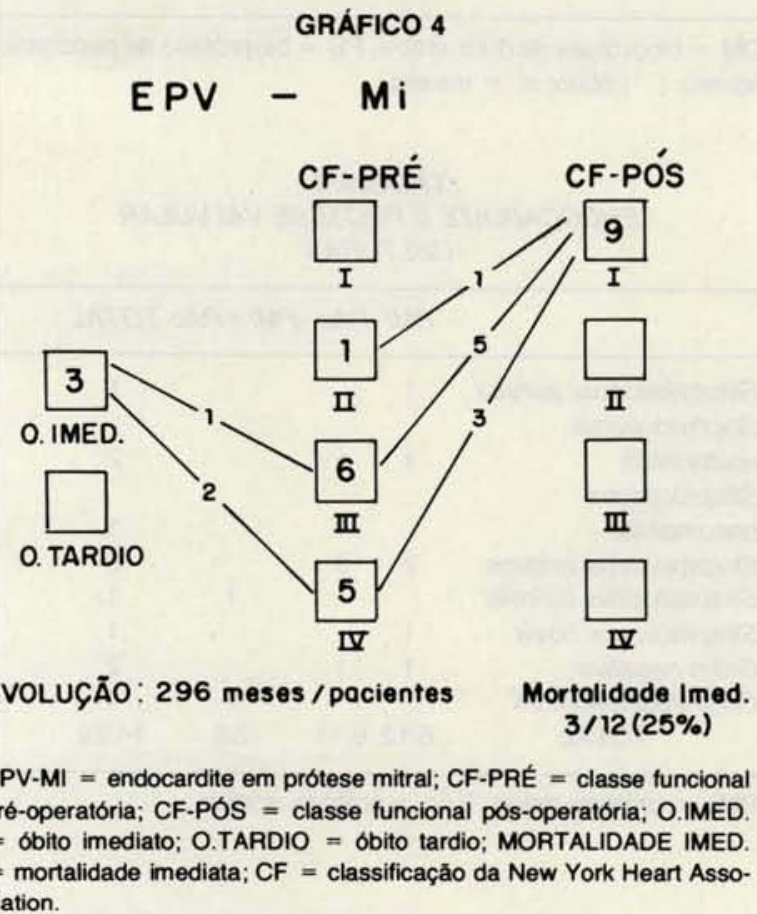

nas EPV mais precoces, e, nas tardias, os estreptococos ${ }^{2}$. Em nossa experiência, a hemocultura foi positiva em $50 \%$ dos casos $(14 / 28)$ e o agente mais comum foi o estreptococo (10/28).

A hemocultura, quando positiva, pode também ter valor prognóstico de acordo com o agente identificado ${ }^{2}$. 4. 9. 12. Constatou-se que as EPV causadas por estreptococos têm mortalidade significativamente menor do que as por estafilococos, enterococos ou agentes gram-negativos.

Não há consenso, na literatura, em relação aos fatores que provocariam a endocardite após o implante da prótese. Nos casos em que a doença se desenvolve precocemente após a cirurgia, a contaminação pré ou 
POMERANTZEFF, P. M. A.; PÊgo-Fernandes, P. M.; KIOKA, Y.; CARdoso, R. H. A.; GaluCCI, S. D. D.; MANSUR, A.; DIAS A. R.; GRINBERG, M.; BITTENCOURT, D.; STOLF, N. A. G.; VERGINELLI, G.; JATENE, A. D. - Tratamento cirúrgico da endocardite em prótese valvular cardiaca. Rev. Bras. Cir. Cardiovasc., 4(1): 43-50, 1989.

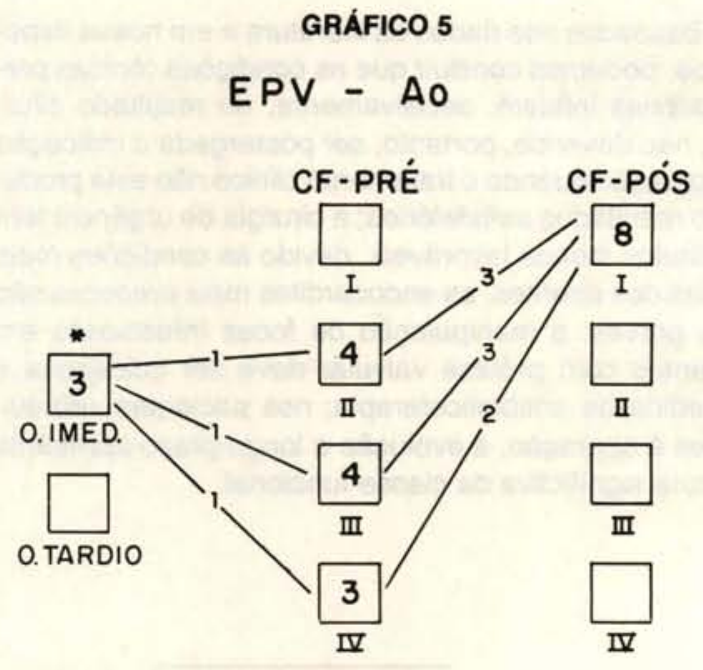

*-Reinfecçõo c/ óbito 32 ? P.O.

EVOLUÇĀO: 300 meses/pacientes Mortalidade Imed. $3 / 11(27.2 \%)$

EPV-AO $=$ endocardite em prótese aórtica; CF-PRE $=$ classe funcional pré-operatória; CF-POS = classe funcional pós-operatória; O.IMED. = óbito imediato; O.TARDIO = obito tardio; MORTALIDADE IMED. $=$ mortalidade imediata; $\mathrm{CF}=$ classificaçăo da New York Heart Association.

\section{GRÁFICO 6}

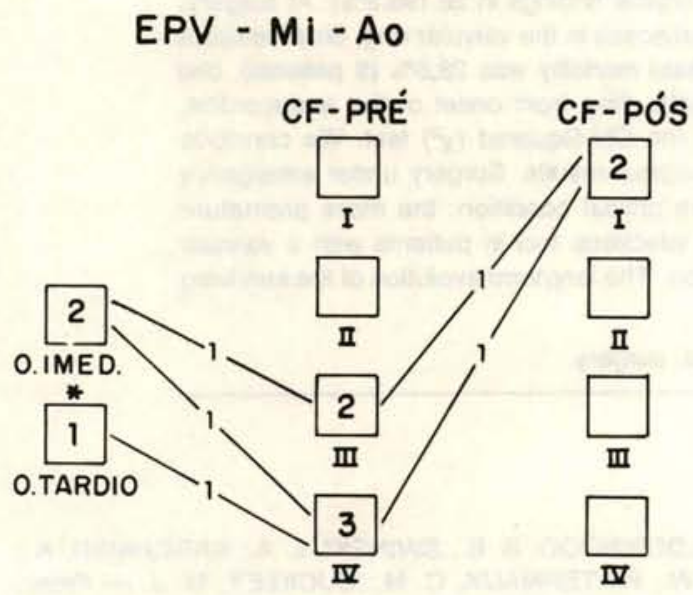

* Reinfecção 3 meses

EVOLUÇÃO: 112 meses/pacientes

Morfalidade Imed. $2 / 5(40 \%)$

EPV-MI-AO $=$ endocardite em prótese mitro-aórtica; $\mathrm{CF}-\mathrm{PRE}=$ classe funcional pré-operatória; CF-POS = classe funcional pós-operatória; O.IMED. = obito imediato; O.TARDIO = obito tardio; MORTALIDADE IMED. $=$ mortalidade imediata; $\mathrm{CF}=$ classificaçăo da New York Heart Association.

intra-operatória da prótese é o fator mais aceito, principalmente quando o agente etiológico é estafilococo ${ }^{3}, 4$, 5, 16. Fatores de risco aventados são os seguintes: substi- tuição valvar aórtica ${ }^{4,16}$, válvulas biológicas ${ }^{11,16}$, válvulas mecânicas ${ }^{3}$, endocardite em valva nativa ou em válvula artificial $^{6,11}$, substituiçōes valvares múltiplas ${ }^{3}$, pacientes do sexo feminino ${ }^{3}$. A maior parte dos nossos pacientes era do sexo masculino e, em 2 deles, havia endocardite na prótese inicialmente substituída. Em nossa experiência, os pacientes que apresentaram EPV pouco tempo após a cirurgia associavam-se a prognóstico pior. Não houve diferença de ocorrência de EPV entre localizações aórtica e mitral, sendo, porém, significativamente mais freqüentes nas duplas trocas. Em 4 doentes, houve manipulação de abscesso, 3 dentários e 1 uterino, previamente ao desenvolvimento da endocardite. Três pacientes apresentavam infecção das vias biliares, 1 broncopneumonia (hemocultura positiva para St. pneumoniae), e 1 diverticulose de cólon (hemocultura revelou St. Bovis ).

O ecocardiograma bidimensional é exame de grande valia no diagnóstico de EPV ${ }^{8}, 10,13,16$. O estudo hemodinâmico não tem sido indicado, rotineiramente, devido ao risco de mobilização de vegetações e a possível piora de função renal.

Nos 6 pacientes em que foi realizado estudo hemodinâmico, havia lesão coronária previamente documentada, revascularização anterior, ou forte suspeita clínica e eletrocardiográfica de coronariopatia. Um paciente com lesão de tronco de coronária esquerda apresentou parada cardíaca no laboratório de hemodinâmica; operado em caráter de urgência, faleceu na sala de cirurgia.

A mortalidade cirúrgica da EPV, apesar da melhoria do diagnóstico e do tratamento, permanece ainda muito alta, variando de $23 \%$ a $64 \%$, $4,5,9,11,13,15,16$, mortalidade esta que é ainda maior quando realizado o tratamento clínico $^{2}, 5,9,11$. Em nossa experiência, a mortalidade do tratamento cirúrgico foi de $28,5 \%$. Os fatores que levam a aumento do risco cirúrgico são a endocardite em prótese logo após a cirurgia de troca valvar ${ }^{4}$, a demora no diagnóstico ${ }^{2,4}$, a insuficiência renal pré- operatória ${ }^{2,4}$, ${ }^{16}$, o agente etiológico não estreptocócico 2 4, 9, 12 , o abscesso do anel ${ }^{5}$, a insuficiência cardíaca severa ${ }^{1}, 4,7$, e a insistência no tratamento clínico quando já existe comprometimento severo do estado geral ${ }^{2}$. Os nossos achados foram condizentes com os encontrados na literatura, sendo que os pacientes com hemoculturas negativas apresentaram mortalidade significativamente maior do que aqueles em que o agente etiológico foi identificado.

Outro fator estatisticamente significativo em nossa casuística foi a mortalidade nos casos de cirurgia de urgência, fato, aliás, explicável pelas condiçōes mais graves dos pacientes.

Não há consenso entre o tipo de válvula a ser utilizado na substituição de uma prótese com endocardite. Alguns autores ${ }^{2}{ }^{16}$ preferem o uso das válvulas biológicas, outros ${ }^{11}$ as mecânicas. Em relação à gravidade da endocardite, alguns autore ${ }^{2}{ }^{2}{ }^{16}$ consideram que, quando ela ocorre em próteses mecânicas, há maiores riscos 
POMERANTZEFF, P. M. A.; PÊgo-Fernandes, P. M.; KIOKA, Y.; CARDOSO, R. H. A.; GaluCCI, S. D. D.; MANSUR, A.; DIAS A. R.; GRINBERG, M.; BITTENCOURT, D.; STOLF, N. A. G.; VERGINELLI, G.; JATENE, A. D. - Tratamento cirúrgico da endocardite em prótese valvular cardiaca. Rev. Bras. Cir. Cardiovasc., 4(1): 43-50, 1989.

de dificuldades no tratamento, e formação de abscesso do anel com maior freqüência. Segundo esses autores, a endocardite em válvulas biológicas pode se limitar ao tecido biológico, sendo mais fácil a esterilização com antibioticoterapia.

Há relativo consenso entre os diversos autores ${ }^{1-16}$, em indicar a cirurgia antes que ocorram compliaçōes sistêmicas. Alguns ${ }^{15}$ recomendam 0 uso da circulação extracorpórea fêmoro-femoral antes da toracotomia e até mesmo antes da anestesia. A antibioticoterapia prolongada, no pós-operatório, também é amplamente utili$z$ zda $^{1-16}$ e o ecocardiograma, além de auxiliar no diagnóstico, é útil no seguimento pós-operatório ${ }^{13}$.
Baseados nos dados da literatura e em nossa experiência, podemos concluir que as condiçōes clínicas préoperatórias influem, decisivamente, no resultado cirúrgico, nāo devendo, portanto, ser postergada a indicaçāo da operação quando o tratamento clínico não está produzindo resultados satisfatórios; a cirurgia de urgência tem resultados menos favoráveis, devido às condiçōes mais críticas dos doentes; as endocardites mais precoces são mais graves: a manipulaçāo de focos infecciosos em pacientes com prótese valvular deve ser cuidadosa $e$ precedida de antibioticoterapia; nos pacientes sobreviventes à operação, a evolução a longo prazo apresenta melhora significtiva da classe funcional.

POMERANTZEFF, P. M. A.; PÊGO-FERNANDES, P. M.; KIOKA, Y.; CARDOSO, R. H. A.; GALUCCI, S. D. D.; MANSUR, A.; DIAS, A. R.; DIAS, A. R.; GRINBERG, M.; BITTENCOURT, D.; STOLF, N A. G.; VERGINELLI, G.; JATENE, A. D. - Surgical treatment of endocarditis in prosthetic valves. Rev. Bras. Cir. Cardiovasc., 4(1): 43-50, 1989.

ABSTRACT: From January/1983 to March/1988, 1512 patients were submitted to valve replacement in the Instituto do Coração, Of these, $28(1.8 \%)$ presented endocarditis in a valvular prosthesis. Seventeen patients were males, their ages varying from 18 to 67 years, with an average and standard deviation of $36.7 \pm 12.9$. The evolution of the functional class (F. C.) revealed 11 patients in (F. C.) IV, 12 in $F$. C. III and 5 in F. C. II (NYHA). Eight $(28.5 \%)$ patients were operated on under emergency conditions. One patient had a mechanical valve and 27 had biological valves. The blood cultures were positive in $14(50 \%)$ patients, the agent most commonly found being Streptococcus viridans (5 cases). Preoperative echocardiogram in 27 patients showed good correlation with the surgical findings in $26(96.2 \%)$. At surgery, 17 patients presented vegetations in the prosthesis and 11 had an abscess in the valvular ring. Bioprosthesis were used as valvular replacement in 27 patients $(96,4 \%)$. Immediate mortality was $28,5 \%$ (8 patients), one death occurring later. An analysis of the association between death, time from onset of the endocarditis, surgical conditions and result of the cultures was carried out by the Chi-Squared $\left(x^{2}\right)$ test. We conclude that the clinical preoperative conditions influence decisively the surgical results. Surgery under emergency conditions has the poorest results because of the patient's more critical condition: the more premature cases of endocarditis are also the more severe. Manipulation of infectious foci in patients with a valvular prosthesis should be careful and preceded by antibiotic administration. The longterm evolution of the surviving patients demonstrated significant functional class improvement.

DESCRIPTORS: endocarditis, surgery; heart valves prosthesis, surgery.

\section{REFERÊNCIAS BIBLIOGRÁFICAS}

1 ARNET, E. N. \& ROBERTS, W. C. - Prosthetic valve endocarditis. Am. J. Cardiol, 38: 281-292, 1976.

2 BAUMGARTNER, W: A.; MILLER, D. C.; EWITZ, B. A.; OYER, P. E.; JAMIESON, S. W.; STINSON, E. B.; SHUMWAY, N. E. - Surgical tieatment of prosthetic valve endocarditis. Ann. Thorac. Surg., 35: 87-101, 1983.

3. CALDERWOOD, S. B.; SWINSKI, L. A.; WATERNAUX, C. M.; KARCHMER, A. W.; BUCKLEY, M. J. - Risk factors of the development of prosthetic valve endocarditis. Circulation, 72: 31-37, 1985.
4 CALDERWOOD, S. B.; SWINSKI, L. A.; KARCHMER, A W.; WATERNAUX, C. M.; BUCKLEY, M. J. - Prosthetic valve endocarditis. J. Thorac. Cardiovasc. Surg., 92: 776-783, 1986.

5 COWGILL, L. D.; ADDONIZIO, V. P.; HOPEMANN, A. R.; HARKEN, A. H. - A practical approach to prosthetic valve endocarditis. Ann. Thorac. Surg., 43: 450-457, 1987 ।

6 D'AGOSTINO, R. S.; MILLER, D. C.; STINSON, E. B.; MITCHELL, R. S.; OYER, P. E.; JAMIESON, S. W.; BALDWIN, J. C.; SHUMWAY, N. E. - Valve replacement in patients with native valve endocarditis: what really determines operative outcome? Ann. Thorac. Surg., 40: 429-437, 1985. 
POMERANTZEFF, P. M. A.; PÊGO-FERnANDES, P. M.; KIOKA, Y.; CARDOSO, R. H. A.; GALUCCI, S. D. D.; MANSUR, A.; DIAS A. R.; GRINBERG, M.; BITTENCOURT, D.; STOLF, N. A. G.; VERGINELLI, G.; JATENE, A. D. - Tratamento cirúrgico da endocardite em prótese valvular cardiaca. Rev. Bras. Cir. Cardiovasc., 4(1): 43-50, 1989.

7 GRAY, I. R. - Infective endocarditis 1937-1987. Br. Heart J., 57: 211-213, 1987.

8 Van HEWERDEN, L. A.; GUSSENHOVEN, E. J.; ROELANDT, J. R. T. C.; HAALEBOS, M. M. P.; MOCHTAR, B.; LIGTOVOET, K. M.; BOS, E. - Intraoperative twodimentional echocardiography in complicated infective endocarditis of the aortic valve. J. Thorac. Cardiovasc. Surg., 93: 587-591, 1987.

9 IVERT, T. S. A.; DISMUKES, W. E.; COBBS, C. G.; BLACKSTONE, E. H.; KIRKLIN, J. W.; BERGDAHL, L. A. - Prosthetic valve endocarditis. Circulation, 69: 223-232, 1984.

10 KINNEY, E. L. \& WEIGHT II, R. J. - Aortic valve vegetations examples of overestimation and underestimation of disease by two-dimentional echocardiography. Am. Heart. J., 113: 1248-1250, 1987.

11 LENGYEL, M. - Risk factors for the development and outcome of prosthetic valve endocarditis. Europ. Heart J., 8(Supl. J): 371-374, 1987.

12 MASUR, H. \& JOHNSON Jr., W. D. - Prosthetic valve endocarditis. J. Thorac. Cardiovasc. Surg., 80: 31-37, 1980.

13 NUÑEZ, L.; LLANA, R.; AGUADO, M. G.; IGLESIAS, A.; LARREA, J. L.; CELEMÍN, D. - Bioprosthetic valve endocarditis: indications for surgical intervention. Ann. Thorac. Surg., 35: 262-270, 1983.

14 FLEISS, J. L. - Statistical methods for rates and proportions. 2.ed. New York, John Wiley \& Sons, 1980.

15 REYCHAUDHURT, T.; CAMERON, E. W. J.; WALBAUM, P. R. - Surgical management of prosthetic valve endocarditis. J. Thorac. Cardiovasc. Surg., 86: 112-114, 1983.

16 ROSSITER, S. J.; STINSON, E. B.; OYER, P. E.; MILLER, D. C.; SCHAPIRA, J. N.; MARTIN, R. P.; SHUMWAY, N. E. - Prosthetic valve endocarditis. J. Thorac. Cardiovasc. Surg., 76: 795-803, 1978.

\section{Discussão}

\section{DR. ISEU AFFONSO DA COSTA} Curitiba, $P R$

Desejamos cumprimentar o Dr. Pomerantzeff e seus colegas, pelo trabalho apresentado e pelos resultados obtidos nesta série de infecções protéticas. $\mathrm{O}$ assunto é de grande importância prática e gravidade, em nosso meio. Em nosso Serviço da Santa Casa de Curitiba e do Hospital de Clínicas, como, certamente, em todos os Serviços cardiocirculatórios do País, o assunto das endocardites desperta grande interesse. Nosso número de casos de infecção em prótese também é grande, respeitadas as proporções da casuística geral. Assim, de junho de 1983 a março de 1989, tivemos 56 casos de endocardite com indicação para operação (8\%), entre 928 pacientes submetidos a substituição valvar. Destes, 13 eram próteses infectadas, o que dá uma incidência de $1,4 \%$, que não está distante da apresentada no trabaIho, de 1,8\%. Gostaria de ouvir comentários do Dr. Pomerantzeff sobre a baixa sensibilidade diagnóstica do exame bacteriológico. Há cerca de 3 anos, ao publicarmos nossos casos de endocardite, tivemos cerca de $70 \%$ de positividade. Cumprimentamos o grupo do InCor, pela baixa mortalidade operatória. Conforme está no texto do trabalho, a mortalidade, na literatura, varia entre 23 e $64 \%$. A mortalidade InCor está, portanto, na faixa inferior daquelas registradas na literatura. Nossa experiência com endocardite em próteses resume-se em: a mortalidade é superior à do InCor (analisando estatisticamente, verificamos que a difereça é significativa); entretanto, como as amostras são pequenas, aplicamos os limites de confiança, às taxas de mortalidade. Os resultados mostram que os limites de confiança da mortalidade chegam a se sobrepor, nas faixas extremas. Pergunto ao Dr. Pomerantzeff se foi possível estabelecer relação entre o tipo de lesão (vegetaçōes, abcessos, fístulas) e mortalidade. Em nosso material, as lesōes simples apresentam mortalidade baixa e muito diferentes das lesōes complicadas.

\section{DR. BERNARDO GARICOCHEA São Paulo, SP}

O Dr. Pablo observou que os seguintes fatores associavam-se a pior prognóstico: o desenvivimento de endocardite precoce após a cirurgia e a insistência no tratamento clínico sem o concurso da cirurgia quando já existe o comprometimento severo no estado geral do paciente. $\mathrm{Na}$ endocardite infecciosa, observam-se 2 fenômenos imunes distintos, um a nível local, outro sistêmico. No sítio da lesão, na vegetação contaminada, a migração de polimorfonucleares e as células "natural killer" têm como objetivo a destruição da lesão, tentativa contínua e infrutífera, já que os mesmos não conseguem penetrar na rede de fibrina que protege os microorganismos. A conseqüência deste ataque à valva acometida é a liberação de linfocinas, mediadoras de resposta inflamatória e radicais livres, que comprometem, progressivamente, áreas sãs perivalvares. A nível periférico, a migração de microorganismos gera uma resposta imune clássica, com o aumento nos níveis circulantes de imunoglobulinas e a formação de imunocomplexos, nem sempre benéficos, é responsável por esplenomegalia, lesão renal, fenômenos articulares e, principalmente, hipocomplementemia, que é um fator fundamental associado à septicemia, nesses pacientes. Nos individuos que desenvolvem lesões precoces pós-operatórias, ou refratárias ao tratamento clínico, geralmente se constatam áreas extensas de depósito de fibrina. Além de albergarem um grande número de microorganismos, essas vegetações geram uma resposta imune local muito itensa, com 
POMERANTZEFF, P. M. A.; PÊGo-FERnANDES, P. M.; KIOKA, Y.; CARDOSO, R. H. A.; GALUCCI, S. D. D.; MANSUR, A.; DIAS A. R.; GRINBERG, M.; BITTENCOURT, D.; STOLF, N. A. G.; VERGINELLI, G.; JATENE, A. D. - Tratamento cirúrgico da endocardite em prótese valvular cardiaca. Rev. Bras. Cir. Cardiovasc., 4(1): 43-50, 1989.

importante reação inflamatória local e depósito de mais fibrina sobre a nova área lesada, que se torna capaz de alojar mais microorganismos, criando um círculo vicioso. Esses pacientes apresentam, portanto, já que, além de possuírem um inóculo bacteriano maior e mais resistente à antibioticoterapia, mostram graus variados de imunodepressão, pelo esgotamento do sistema imune, constantemente estimulado, e pela queda rápida do estado nutricional. No grupo de pacientes descrito pelo $\mathrm{Dr}$. Pablo, notamos que o número maior de complicaçōes (abscessos de anel valvar, aneurisma no seio de Valsalva, perfuração valvar) se associa com a piora progressiva do estado geral e do prognóstico dos mesmos. A deterioração do perfil imune destes indivíduos torna este estado pouco responsivo à antibioticoterapia e à abordagem puramente clínica, sendo imperativa a remoçāo cirúrgica precoce do agente que perpetua o processo infeccioso à vegetação valvar. Portanto, não acreditamos ser válido postergar o procedimento cirúrgico em pacientes com quadros de endocardite instalada precocemente em próteses e em vegetaçōes documentadamente extensas, mesmo que esses individuos não apresentem níveis de complemento muito baixos, ou de imunocomplexos muitos altos. Muito obrigado.

\section{DR. POMERANTZEFF (Encerrando)}

Agradecemos os comentários e, respondendo ao Dr. Iseu, gostariamos de salientar que acreditamos que existe uma grande influência sobre a mortalidade, o momento da indicação cirúrgica. Nem sempre é fácil saber qual é esse momento ideal, mas devemos estar atentos para detalhes que indiquem uma deterioração do paciente. Como foi mostrado na apresentação do trabalho, houve maior mortalidade quando o aparecimento da endocardite, após o implante de prótese, foi precoce e, nos casos em que existem abscessos de anel, a gravidade é, sem dúvida, maior. Acreditamos, também, que a relação gravidade/tipo de germe deve ser levada em conta. Os pontos abordados pelo Dr. Bernardo são, sem dúvida, fundamentais e explicam muito bem o porque de não podermos esperar demais para indicarmos a cirurgia em pacientes com endocardite em prótese. 